\title{
Role of Nrf2 and Autophagy in Acute Lung Injury
}

\author{
Montserrat Rojo de la Vega ${ }^{1} \cdot$ Matthew Dodson $^{1} \cdot$ Christine Gross $^{2} \cdot$ Heidi M. Mansour ${ }^{3}$. \\ R. Clark Lantz ${ }^{1,4}$ Eli Chapman ${ }^{1,5}$ - Ting Wang ${ }^{6}$ - Stephen M. Black ${ }^{2}$. \\ Joe G. N. Garcia ${ }^{6}$ • Donna D. Zhang ${ }^{1,5}$
}

Published online: 6 February 2016

(C) Springer International Publishing AG 2016

\begin{abstract}
Acute lung injury (ALI) and acute respiratory distress syndrome (ARDS) are the clinical manifestations of severe lung damage and respiratory failure. Characterized by severe inflammation and compromised lung function, ALI/ ARDS result in very high mortality of affected individuals. Currently, there are no effective treatments for ALI/ARDS, and ironically, therapies intended to aid patients (specifically mechanical ventilation, MV) may aggravate the symptoms. Key events contributing to the development of ALI/ARDS are increased oxidative and proteotoxic stresses, unresolved inflammation, and compromised alveolar-capillary barrier function. Since the airways and lung tissues are constantly exposed to gaseous oxygen and airborne toxicants, the
\end{abstract}

This article is part of the Topical Collection on Free Radicals Medicine

Stephen M. Black

steveblack@email.arizona.edu

Joe G. N. Garcia

skipgarcia@email.arizona.edu

$\triangle$ Donna D. Zhang

dzhang@pharmacy.arizona.edu

1 Department of Pharmacology and Toxicology, University of Arizona, Tucson, AZ, USA

2 Department of Medicine, Division of Translational and Regenerative Medicine, University of Arizona, Tucson, AZ, USA

3 Skaggs Pharmaceutical Sciences Center, University of Arizona, Tucson, AZ, USA

4 Department of Cellular and Molecular Medicine, University of Arizona, Tucson, AZ, USA

5 Arizona Cancer Center, University of Arizona, Tucson, AZ, USA

6 Arizona Respiratory Center and Department of Medicine, University of Arizona, Tucson, AZ, USA bronchial and alveolar epithelial cells are under higher oxidative stress than other tissues. Cellular protection against oxidative stress and xenobiotics is mainly conferred by $\mathrm{Nrf} 2$, a transcription factor that promotes the expression of genes that regulate oxidative stress, xenobiotic metabolism and excretion, inflammation, apoptosis, autophagy, and cellular bioenergetics. Numerous studies have demonstrated the importance of Nrf2 activation in the protection against ALI/ARDS, as pharmacological activation of Nrf2 prevents the occurrence or mitigates the severity of ALI/ARDS. Another promising new therapeutic strategy in the prevention and treatment of ALI/ARDS is the activation of autophagy, a bulk protein and organelle degradation pathway. In this review, we will discuss the strategy of concerted activation of Nrf2 and autophagy as a preventive and therapeutic intervention to ameliorate ALI/ARDS.

Keywords Acute lung injury · Oxidative stress · Nrf2 . Autophagy

\section{Acute Lung Injury and Acute Respiratory Distress Syndrome}

Acute lung injury (ALI) and its more severe form, acute respiratory distress syndrome (ARDS), are severe inflammatory diseases of the lung caused by disruption of the lung endothelial (capillaries) and epithelial (alveoli) barriers. ARDS was first described by Ashbaugh et al. in [3], but it was not until 1994 that a consensus definition and description of ALI/ ARDS was achieved by the American-European Consensus Conference (AECC) [3]. The clinical criteria that define ALI/ ARDS are the following: (1) acute onset of respiratory symptoms, (2) chest radiographs with bilateral infiltrates, (3) a ratio of partial pressure of oxygen in arterial blood $\left(\mathrm{PaO}_{2}\right)$ to 
fraction of inspired oxygen $\left(\mathrm{FiO}_{2}\right)$ of $<200 \mathrm{mmHg}$ for ARDS and $<300 \mathrm{mmHg}$ for ALI, (4) pulmonary capillary wedge pressure (PCWP) of less than $18 \mathrm{mmHg}$, and (5) the absence of clinical evidence of primary left atrial hypertension [7].

It is estimated that $64.2-78.9$ per 100,000 persons per year develop ALI, which has a mortality rate of $38.5 \%$ [95]. Based on these data, approximately 190,600 people in the USA each year will develop ALI, and 74,500 people will not survive [82, 95]. Some survivors recover completely [35, 95]; however, others may have lasting damage to their lungs and develop additional health problems $[62,95]$.

\section{Pathogenesis of ALI/ARDS}

As ALI/ARDS is a syndrome, or a collection of signs and symptoms, and not a specific disease, there are many insults that can produce lung injury directly or indirectly and cause ALI/ARDS [7]. Direct pulmonary injury involves a disease process that begins in the lungs by causing primarily lung damage, such as lung contusions, diffuse pulmonary infections (bacterial, viral, and fungal pneumonia), aspiration of gastric contents, near drowning, inhalation of toxic substances, or hyperoxia [7]. ALI/ARDS can be induced or aggravated by mechanical ventilation (MV), a therapeutic intervention used to assist patients with compromised respiratory functions due to edema, trauma, or general anesthesia [101]. MV causes ventilation-induced lung injury (VILI) due to different types of lung injury, including (1) volutrauma, from lung over-distension; (2) barotrauma, from the direct effect of high pressure on the lung; (3) atelectrauma, from the shear stress of repetitive opening and closing of alveoli; and (4) biotrauma, from the generation of cytokines and inflammatory cascades [86]. Indirect pulmonary injury is a systemic disease process that affects the lungs secondarily, as in sepsis syndrome and systemic inflammatory response syndrome (SIRS), severe non-thoracic trauma, shock, acute pancreatitis, cardiopulmonary bypass, transfusion-related ALI (TRALI), disseminated intravascular coagulation (DIC), and burns [7].

Direct or indirect injury to the lung parenchyma leads to damage of type I and type II epithelial cells of the alveoli, and of endothelial cells of the pulmonary capillaries, resulting in loss of the alveolar-capillary barrier [6]. Without this barrier, fluid from the capillaries leaks into the interstitium and the alveoli, causing pulmonary edema, collapse of the lungs (atelectasis), and respiratory failure [6]. These classic histological manifestations of ARDS in the lung are called diffuse alveolar damage (DAD) [6]. The pathogenesis of DAD begins with an acute/exudative stage ( $\leq 7$ days after insult), characterized by damage to the alveolar-capillary barrier, resulting in the formation of an exudate in alveolar spaces and interstitium, activation of resident alveolar macrophages, and release of pro-inflammatory cytokines to promote neutrophil infiltration. Macrophages and neutrophils secrete various proteases and oxidants that further injure alveolar endothelial and epithelial cells and digest the alveolar stroma. As a result, type II alveolar epithelial cells and fibroblasts proliferate in an attempt to repair the lung injury during the organizing/ fibroproliferative stage [6]. DAD may gradually resolve within 6-12 months; however, the most common outcome is a fibrotic stage that results in chronic respiratory compromise $[6,64]$.

\section{Experimental Models for Studying ALI/ARDS In Vivo}

Based on the diverse etiology of ALI/ARDS, many different modeling strategies have been developed in animals in an attempt to reproduce the features of human ALI/ARDS. Direct lung injury in animals can be induced by (1) the intratracheal or intranasal administration of bacteria or bacterial products, such as lipopolysaccharide (LPS) from the outer cell wall of gram negative bacteria, or peptidoglycan and lipoteichoic acid from gram positive bacteria, to reproduce diffuse pneumonia; (2) the administration of hydrochloric acid or gastric particulates to reproduce aspiration; (3) the administration of high inspired fractions of oxygen to induce hyperoxia; or (4) the induction of ischemia/reperfusion by clamping the hilum [65]. Indirect lung injury is based on (1) reproducing sepsis using cecal ligation and puncture, (2) the administration of intravenous bacteria or LPS, or (3) mesenteric ischemia/reperfusion [65]. Animal models of VILI consist of mechanical ventilation with high tidal volumes and low positive end-expiratory pressure [100].

\section{Current Therapeutic and Treatment Options for ALI/ARDS}

The treatment for ARDS is mainly supportive and focuses on maintaining ventilation and oxygenation, normal cardiac function, and nutritional support, as well as the administration of nitric oxide (NO) and corticosteroids, and the prevention of further complications $[64,99]$. A more appealing strategy would be to treat the underlying cause for the lung injury and respiratory distress $[64,99]$. Central to the pathogenesis of ALI/ARDS is the generation of reactive oxygen species (ROS) that cause oxidative stress. Multiple cell types in the lungs, including endothelial cells, neutrophils, eosinophils, alveolar macrophages, and alveolar epithelial cells, are major ROS generators [67]. ROS may be produced by inflammatory cells (macrophages and neutrophils) trying to fight infections, or by dysfunctional mitochondria or enzymatic systems in alveolar epithelial cells and capillary endothelial cells after lung tissue damage by hyperoxia, electrophilic xenobiotics, 
and shear stress $[8,9,79,97]$. Antioxidants have been shown to reduce the severity of ALI/ARDS in multiple mouse models that use different initiating insults, highlighting the central role of ROS in the pathophysiology of ALI/ARDS $[2,10,36-38,56,85,103,104,106,112]$.

\section{The Nrf2 Signaling Pathway}

Nrf2 (nuclear factor erythroid-2 related factor 2) is a transcription factor that regulates an adaptive cellular defense response to various stresses, including oxidative, proteotoxic, and metabolic stresses, as well as inflammation (Fig. 1). Nrf2 heterodimerizes with small Maf (sMaf) proteins and together they bind the antioxidant response elements (AREs) in the regulatory regions of Nrf2 target genes that participate in the regulation of oxidative stress (HO-1, GCLM, TXNRD1), xenobiotic metabolism and excretion (NQO1, AKR1C1, GSTA, $M R P 1)$, inflammation ( $T G F-\beta, N F-\kappa B)$, apoptosis ( $B c l-2, B c l$ $x L$ ), autophagy (p62), and cellular bioenergetics (G6PD, $P P A R \gamma$ ) (Fig. 1) [29, 42, 47]. Even though Nrf2 is ubiquitously expressed in all cell types and tissues, its protein levels are kept low under basal (homeostatic) conditions since it is constantly degraded. The main negative regulator of $\mathrm{Nrf} 2$ is Keap1 (Kelch-like ECH associated protein 1), a substrate adaptor for a Cullin 3 (Cul3)-containing E3 ubiquitin ligase complex that ubiquitinates Nrf2 to promote its degradation by the $26 \mathrm{~S}$ proteasome $[48,94,108]$. Nrf2 binds through its $\mathrm{N}$ terminal ETGE and DLG motifs to two Keap1 proteins at the Kelch domain in what is known as the hinge-and-latch model: the ETGE (hinge) binds to the Kelch domain in Keap1 more strongly than the DLG (latch) motif, which oscillates between open and closed conformations [5, 92, 93]. Nrf2 ubiquitination occurs only when both of its motifs are binding to Keap1 in a closed conformation [5]. Keap1 is a sensor of the intracellular redox status: oxidants and electrophilic chemopreventive compounds covalently modify several important cysteine residues, in particular cysteine 151 (C151), in Keap1. Oxidation or adduction of C151 causes a conformational change in Keap1 that affects the DLG-Kelch interaction, thus preventing Nrf2 ubiquitination and subsequent degradation [109, 110]. Consequently, Nrf2 protein is stabilized, accumulates, and translocates into the nucleus to activate the transcription of its target genes. Many known electrophilic chemopreventive compounds activate Nrf2 signaling through the canonical mechanism, and mutation of cysteine 151 to serine (Keap1-C151S) in Keap1 completely abolishes Nrf2 upregulation by canonical Nrf2 activators [42]. Signal termination occurs when the redox balance is restored, or when the chemopreventive compounds are metabolized and eliminated. At this point, Keap1 shuttles into the nucleus, binds to Nrf2, and brings it back into the cytosol for degradation [87].

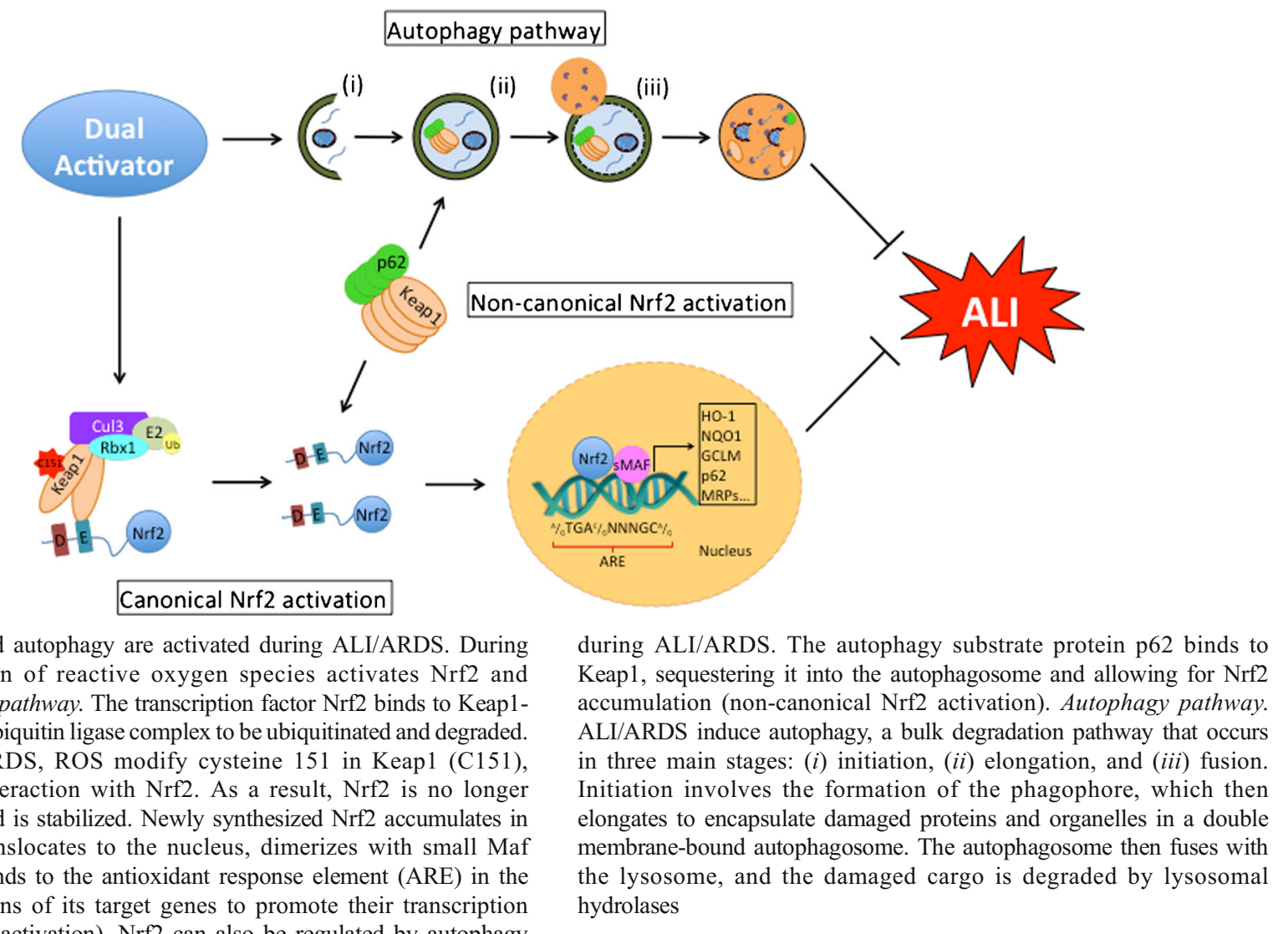

Fig. 1 Nrf2 and autophagy are activated during ALI/ARDS. During ALI, production of reactive oxygen species activates Nrf2 and autophagy. Nrf2 pathway. The transcription factor Nrf2 binds to Keap1Cul3-Rbx 1 E3 ubiquitin ligase complex to be ubiquitinated and degraded. During ALI/ARDS, ROS modify cysteine 151 in Keap1 (C151), affecting its interaction with $\mathrm{Nrf} 2$. As a result, $\mathrm{Nrf} 2$ is no longer ubiquitinated and is stabilized. Newly synthesized Nrf2 accumulates in the cytosol, translocates to the nucleus, dimerizes with small Maf proteins, and binds to the antioxidant response element (ARE) in the regulatory regions of its target genes to promote their transcription (canonical Nrf2 activation). Nrf2 can also be regulated by autophagy during ALI/ARDS. The autophagy substrate protein $\mathrm{p} 62$ binds to Keap1, sequestering it into the autophagosome and allowing for Nrf2 accumulation (non-canonical Nrf2 activation). Autophagy pathway. ALI/ARDS ind three autophagy, a bulk degadation pathway that occurs hydrolases 


\section{The p62-Dependent, Non-canonical Mechanism, of Nrf2 Regulation}

Recent studies have identified other Nrf2 regulatory protein complexes that participate in other pathophysiological processes $[27,73,74,102]$. One of these corresponds to the autophagy-related protein p62 (sequestosome 1, SQSTM1), which activates Nrf2 [52]. Autophagy is a bulk degradation pathway that involves the sequestration of damaged cellular components into an autophagosome, which fuses with the lysosome to form an autolysosome that degrades the cargo using lysosomal enzymes (see below for a more detailed description of the pathway and its role in ALI). p62 is an adaptor protein that recognizes the degradation cargo and brings it to the autophagosome; importantly, p62 is itself an autophagy degradation target. Autophagy inhibition at the late stage causes accumulation of autophagosomes and results in pathological activation of Nrf2 signaling [30, 41, 49, 52, 81]. This occurs because p62 contains a pSTGE motif that competes with the ETGE/DLG motifs in Nrf2 for Keap1 binding [39, 41]. When the number of autophagosomes increases, p62 sequesters Keap1 and thus Nrf2 is stabilized (Fig. 1) [44]. Our group called this mode of Nrf2 activation the non-canonical mechanism, as it is Keap1-C151-independent but p62dependent [52]. For example, arsenic, a human carcinogen, is an Nrf2 activator that induces Nrf2 through blockage of autophagy-lysosomal fusion [53]. This mode of Nrf2 activation arises from a pathological state (autophagy dysregulation) and results in prolonged Nrf2 activation (the "dark side" of $\mathrm{Nrf2);} \mathrm{therefore,} \mathrm{it} \mathrm{is} \mathrm{not} \mathrm{desirable} \mathrm{for} \mathrm{cellular} \mathrm{protection.} \mathrm{On}$ the other hand, induction of autophagy (increased autophagy flux) leads to the sequestration of Keap1-p62 complexes into autophagosomes, resulting in lysosomal-mediated degradation of Keap1 and controlled Nrf2 activation. This mode of Nrf2 activation is intermittent and confers protection. Nrf2 also promotes the ARE-driven expression of p62, indicating that autophagy and the Nrf2-mediated antioxidant response work in concert to restore cellular homeostasis [89].

\section{The Protective Role of Nrf2 in ALI}

The airway and lung epithelial cells constitute an important interphase between the body and the environment, and as such, these tissues are constantly exposed to oxidative stress and toxicants [22]. Therefore, the expression of intra and extracellular antioxidants in airway epithelial cells and fluids is prominent. Numerous in vitro and in vivo studies have demonstrated the importance of $\mathrm{Nrf} 2$ activation to decrease oxidative stress and inflammation in conditions such as pulmonary fibrosis, cystic fibrosis, emphysema, COPD, ALI, asthma, bronchopulmonary dysplasia, and airway infections $[12,23]$. In the particular context of ALI/ARDS, the benefits of both basal and induced Nrf2 signaling have been investigated in various cellular and mouse models, as well as in human patient samples, as will be discussed below.

As mentioned above, the main regulation of Nrf2 occurs post-transcriptionally; however, over 500 single nucleotide polymorphisms (SNPs) in the regulatory and coding regions of Nrf2 have been identified [18]. The first evidence that Nrf2 had a role in ALI came from identification of $N r f 2$ as a candidate gene for ALI susceptibility by positional cloning in mice and subsequent analysis of SNPs in human populations and in vitro functional studies [63]. Multiple studies have determined that SNPs in Nrf2 confer susceptibility to ALI and other respiratory diseases like COPD and asthma [20, $24,28]$. In a hyperoxia-induced ALI mouse model, the functional effects of promoter and coding SNPs revealed that some haplotypes characteristic to certain inbred mouse strains confer increased susceptibility to hyperoxia due to lower $\mathrm{Nrf} 2$ mRNA expression and compromised protein function [20]. These studies will help identify populations at greater risk of developing ALI that would benefit from more effective chemopreventive interventions through $\mathrm{Nrf} 2$ upregulation.

Hyperoxia-induced ALI causes oxidative lung damage. $\mathrm{Nrf}^{-1-}$ mice were more susceptible to hyperoxia (95-98\% oxygen, $72 \mathrm{~h}$ ) and developed more severe ALI-like phenotypes than $\mathrm{Nrf}^{+/+}$mice, as determined by increased lung permeability, inflammation, and lung epithelial damage [21]. In $\mathrm{Nrf} 2^{-/}$ mice, both the basal and induced expression of Nrf2 target genes were lower than in $\mathrm{Nrf}^{+/+}$, demonstrating that $\mathrm{Nrf} 2$ transcriptional activity is key in the response to hyperoxia-induced ALI. Furthermore, conditional deletion of Nrf2 in club cells (Clara cells, secretory bronchoepithelial cells) using cell typespecific Cre recombinase $\left(C C S P-C r e \times N r f 2^{f l l f l}\right)$ yielded mice more sensitive to hyperoxia-induced ALI, confirming that Nrf2 activity in the airways is necessary for protection in this model system [77]. Additionally, unresolved oxidative stress and inflammation in $\mathrm{Nrf}^{-/-}$mice increased apoptosis in sublethal hyperoxic exposure ( $48 \mathrm{~h}$ ) and compromised tissue repair [76]. These results indicate that $\mathrm{Nrf2}$ has additional roles that go beyond the resolution of oxidative stress, including the regulation of inflammation and tissue remodeling factors [23]. Currently, it is unknown if Nrf2 modulates these processes directly or indirectly, so further detailed mechanistic studies are needed to elucidate these Nrf2-dependent effects. Pharmacological Nrf2 activation as a strategy to prevent and treat hyperoxia-induced ALI has been explored. The Nrf2 activator CDDO-Im (a synthetic triterpenoid compound, CDDOimidazole) conferred resistance against hyperoxia [78]. In contrast to the clear effects of $\mathrm{Nrf} 2$ upregulation, administration of direct antioxidants like N-acetylcysteine (NAC), has limited efficacy in protection against hyperoxia-induced ALI. Administration of antioxidants to quench ROS is a strategy with limited effectiveness due to their limited availability and the fact that once oxidized they are useless, and some antioxidants only 
scavenge certain ROS selectively. However, Nrf2 activation promotes sustained expression of endogenous antioxidants, detoxifying/excretion enzymes, repair and degradation proteins, and metabolic reprogramming [29]. Together, these Nrf2 downstream effector proteins remove insults, neutralize ROS, and repair damage to restore cellular homeostasis. In addition, Nrf2 regulates the expression of anti-inflammatory, pro-proliferative, anti-apoptotic, and autophagy-related genes, constituting a more holistic approach to counteract and repair the damage [42]. As such, Nrf2 activation provides not only short-term beneficial effects but also confers medium and longterm protection.

In high tidal volume MV models that induce VILI/ALI, $\mathrm{Nrf}^{-/-}$mice have greater structural damage, increased alveolar-capillary permeability, greater inflammation and oxidative stress than $\mathrm{Nrf}^{+/+}$mice [71]. Supplementation of NAC decreased VILI in $\mathrm{Nrf2}^{-/-}$mice, indicating that oxidative stress is a major contributing factor in the pathogenesis of VILI. Subsequent studies have been performed to evaluate the effects of Nrf2 activation in the protection against VILI. Sodium sulfide protects against VILI by upregulating Nrf2 target genes involved in the restoration of redox balance [31]. Our group recently demonstrated that a newly identified Nrf2 activator, the carotenoid and food additive bixin, protects against VILI only in $\mathrm{Nrf}^{+/+}$but not $\mathrm{Nrf2} 2^{-/-}$mice [90].

ALI-like symptoms can also be chemically induced in mice using agents that cause oxidative damage and inflammation. The phenolic antioxidant butylated hydroxytoluene (BHT) is used as food additive to prevent the oxidation of lipids, and while it is relatively safe in humans, it is toxic when administered to mice. Oral administration of BHT is noxious to alveolar type I epithelial cells, the cells that comprise most of the alveolar structure and are responsible for the gas exchange and barrier functions [98]. BHT causes ALI-like symptoms in the lungs of $\mathrm{Nrf2}^{+/+}$and $\mathrm{Nrf2} 2^{-/}$mice, which develop massive edema and hemorrhage; however, $\mathrm{Nrf}^{-/-}$mice are much more susceptible [12]. The precise cytotoxic mechanism of BHT in the lung has not been clarified, but oxidative stress may play a central role as $\mathrm{Nrf}^{+/+}$mice are more resistant [25]. The effects of oxidative lung damage have been studied in an ozone $\left(\mathrm{O}_{3}\right)$ mouse model. $\mathrm{O}_{3}$ is a strong oxidant and air pollutant that greatly affects the airways and lungs. Predictably, Nrf2 deficiency in mice exacerbates oxidant-induced lung injury [19]. An important implication of this study is that Nrf2 activation by chemopreventive compounds could be very beneficial for populations living in areas with poor air quality exposed to very high $\mathrm{O}_{3}$. Carrageenin, a seaweed-derived polysaccharide used as a thickening and emulsifying agent in food and pharmaceuticals, induces acute inflammation and ALI-like symptoms after intratracheal injection [68]. Consistently, $\mathrm{Nrf}^{-1-}$ mice are more susceptible to carrageenininduced ALI than $\mathrm{Nrf2}^{+/+}$mice [68]. This study also helped to elucidate the anti-inflammatory functions of Nrf2.
Carrageenin-induced inflammation induced COX-2 (cyclooxygenase 2), an enzyme that converts arachidonic acid into prostaglandins, which are mediators of inflammation [80]. In particular, 15-deoxy- $\Delta^{12,14}$-prostaglandin $\mathrm{J}_{2}\left(15 \mathrm{~d}-\mathrm{PGJ}_{2}\right)$ activates $\mathrm{Nrf}$, and pharmacological inhibition of COX-2 induced severe ALI in $\mathrm{Nrf}_{2} 2^{+/+}$mice comparable to that of $\mathrm{Nrf}^{-/-}$, which may be due at least in part to decreased expression of Nrf2 target genes $[68,80]$. These results indicate that resolution of inflammation and oxidative injury are both important to prevent ALI.

Extensive work has been performed to characterize the biological effects of acute exposure to certain gases that represent particularly important hazards, either for their use as chemical warfare agents or due to common work place exposure. These studies have demonstrated that lung epithelial cells are among the first to be exposed to toxic gases such as chlorine $\left(\mathrm{Cl}_{2}\right)$, phosgene, mustard gas, and hydrogen sulfide $\left(\mathrm{H}_{2} \mathrm{~S}\right)$, making these cells a particularly important target for toxicity and for therapeutic intervention $[13,72]$. A common feature of these gases is that acute exposure causes airway and lung epithelial cell apoptosis, alveolar-capillary barrier dysfunction, and an exacerbated inflammatory response [66]. Central to the pathophysiological mechanisms of these gases is the generation of ROS [113]. Many studies have proposed the use of antioxidants to diminish the effects of acute exposure to toxic gases as no effective antidotes or countermeasures have yet been discovered [1, 13, 43, 72]. Antioxidants like NAC and the metalloporphyrin AEOL10150 have been used with moderate success to reduce ROS, oxidative damage, and inflammation $[43,66]$. Few studies have investigated the beneficial effects of targeted Nrf2 activation to ameliorate toxic gas exposure, but this strategy may prove to be more effective.

Nrf2 also participates in infection-induced ALI. The bacteria Staphylococcus aureus causes pneumonia and in extreme cases progresses to ALI due to increased alveolar permeability, neutrophil infiltration, and cytokine production. $\mathrm{Nrf}^{-/-}$ mice developed ALI after $S$. aureus inoculation, unlike $\mathrm{Nrf}^{+/+}$mice, due to enhanced inflammation and decreased mitochondrial biogenesis in alveolar cells of $\mathrm{Nrf2}^{-/-}$mice [4]. Mitochondria not only maintain cellular energy production, but when damaged, they can also be a major source of ROS. Autophagy, in particular mitophagy, degrades damaged mitochondria. Therefore, these results suggest that the mitochondrial effects of Nrf2 and possibly its crosstalk with mitophagy are essential for lung adaptation and recovery from injury [29].

\section{The Autophagy-Lysosomal Pathway}

The autophagy-lysosomal pathway is a key cellular degradation pathway responsible not only for the removal of longlived proteins, but also damaged or dysfunctional proteins 
and organelles, breaking them down to monomeric components for re-use by the cell. Autophagic dysfunction has been linked to the progression of a number of diseases including diabetes, neurodegeneration, cardiovascular disease, pulmonary disease, and cancer, demonstrating the importance of proper autophagic function in preserving normal cellular homeostasis [26]. Three main autophagic pathways have been identified: microautophagy, chaperone-mediated autophagy (CMA), and macroautophagy (hereafter referred to as autophagy). The three main stages of the autophagy pathway are, (i) initiation, (ii) elongation, and (iii) fusion (Fig. 1), and are mediated by a number of tightly regulated protein-protein and protein-lipid interactions. Initiation of autophagy requires formation of the Beclin-1/Vps34/UVRAG/Atg14L complex, which based on its binding partners, can result in either autophagy activation (i.e., bound to AMBRA-1 or Bif-1) or autophagy inhibition (i.e., bound to Rubicon or GAPR-1) [50, 55]. Following initiation, elongation of the phagophore involves the activation of two autophagy-related (Atg) proteindependent ubiquitin-like conjugation systems, Atg5 conjugation to Atg12 mediated by Atg7 and Atg10, and the conjugation of microtubule-associated protein light chain 3 (LC3) to phosphatidylethanolamine via Atg3 and Atg10 [75]. The elongating phagophore encapsulates its cargo to form a double membrane-bound autophagosome, which then fuses with the lysosome via the interaction between endosomal sorting complexes required for transport (ESCRT) proteins, soluble Nethylmaleimide-sensitive factor attachment receptor (SNARE) proteins, as well as Rab7 and a number of vacuolar protein sorting (Vps) proteins [75]. This fused vesicle, termed the autolysosome, can then degrade its contents using a battery of hydrolases, which break down the cargo for re-use by the cell.

\section{Activation of Autophagy During ALI}

While the role of autophagy in chronic lung disease and cigarette smoke exposure has been studied [16, 83], very few studies have investigated the role of autophagy in ALI. As mentioned above, ALI is induced by different stimuli, including mechanical injury, exposure to industrial or environmental toxicants, infection, and sepsis. Autophagy has been shown to contribute to the progression of acute viral and bacterial lung infection. $\operatorname{Atg} 5^{\text {fllffl }} ; L y s M-C r e^{+}$mice, which have autophagy deficient macrophages, exhibit increased bacterial burden and inflammation following infection with Mycobacterium tuberculosis [11]. Autophagy also plays a key role in alveolar macrophages and mast cells in response to Pseudomonas aeruginosa infection, with knockdown of Beclin1 or Atg5, two key autophagy initiation proteins, resulting in decreased clearance and intracellular accumulation of Pseudomonas in infected macrophages [107]. These studies indicate that autophagy can play either a protective or detrimental role, depending on the pathogen and cell type affected during lung infection.

One of the main initiators of autophagy in ALI is increased oxidative stress, which has been shown to induce autophagy in a number of lung cell lines and animal models of lung injury [60]. For example, lung endothelial cells of rodents exposed to sulfur mustard or nitrogen mustard exhibit increased oxidative stress and autophagy activation within $6 \mathrm{~h}$ of gas exposure [61]. $\mathrm{O}_{3}$ exposure has also been shown to result in oxidative stress-induced autophagy in the rat lung [88]. Heavy metal exposure, such as arsenic trioxide, can also lead to oxidative stress and autophagy dysregulation in human bronchial epithelial cells [111]. Exposure of H441 lung epithelial cells to chlorine gas $\left(\mathrm{Cl}_{2}\right)$ induces mitochondrial dysfunction and increased reactive oxygen species production $1 \mathrm{~h}$ after exposure, with increased autophagy being associated with improved bioenergetic function by $6 \mathrm{~h}$ post-exposure [46]. While autophagy upregulation is thought to be sufficient for preventing toxic protein aggregation during mild oxidative stress, if the damage is too great and autophagy can no longer compensate, apoptosis is initiated leading to cell death [17]. This may account for why autophagy upregulation is generally observed during lung injury, but is not always sufficient to mitigate or protect against inflammatory damage. Thus, a need for a better understanding of the temporal regulation of autophagy activation during ALI will be required to create therapeutics that maintain the beneficial effects of autophagy upregulation and reduce proteotoxic effects following toxic-gas exposure, and in turn, preserve lung function.

\section{Protective Role of Autophagy During ALI}

Studies have also demonstrated that upregulation of autophagy can be protective during ALI. For example, pharmacological activation of autophagy using rapamycin, a wellestablished autophagy activator, enhances the clearance of Pseudomonas from lung epithelial cells, whereas inhibition of autophagy using the lysosomal inhibitor chloroquine resulted in the accumulation and decreased killing of Pseudomonas bacteria [45]. Autophagy is downregulated following cecal ligation and puncture (CLP) injury, a model for sepsisinduced ALI, with rapamycin treatment enhancing autophagic activity, reducing the pro-inflammatory response, and preventing activation of the apoptotic cascade in septic mice [105]. Transgenic mice overexpressing $L C 3$, a protein integral for autophagosome formation, also exhibit improved survival following CLP [57]. LPS-induced lung damage is exacerbated by the inhibition of autophagy, and rapamycin treatment decreased LPS-induced inflammatory cytokine production and secretion in lung macrophages $[32,58,69]$. Mice administered trehalose (2 \% in the drinking water), an mTOR-independent 
autophagy activator, for 6 weeks or aerosolized trehalose administration for $24 \mathrm{~h}$ prior to $\mathrm{Cl}_{2}$ exposure demonstrated improved bioenergetic function and decreased lung inflammation [46]. Rapamycin treatment following paraquat-induced oxidative stress and ALI also significantly reduced lung inflammation and damage in mice via the inhibition of mTOR to activate autophagy [14]. Interestingly, low dose carbon monoxide has also been shown to stimulate oxidative stress, which upregulates autophagy to protect against hyperoxia-induced lung injury [54]. These studies indicate that autophagy activation, both before and after toxic insult, can significantly decrease lung inflammation, improve lung cell bioenergetic function, and mitigate the damage associated with ALI.

\section{Dual Activation of Nrf2 and Autophagy as a Potential Therapy for ALI}

As the above studies indicate, proper upregulation of Nrf2 and the autophagy pathway are required to mitigate damage during ALI, and thus represent two key pathways that can be targeted for therapeutic benefit. Since there is crosstalk between autophagy and Nrf2 activation through the Keap1-p62 interaction, it is presumable that dual pathway activators could be used to maximize the therapeutic benefit of Nrf2 and autophagy activation, however, the mechanism of activation must be considered.

In recent years, uncontrolled Nrf2 activation, the "dark side" of Nrf2, has been recognized as a contributing factor of cancer chemoresistance and tissue damage $[44,51]$. This uncontrolled activation may result in either constitutive or prolonged activation of Nrf2. The constitutive activation of Nrf2 observed in numerous cancer cell lines and patient tumor samples confers them a more malignant phenotype, as assessed by higher proliferative rates, increased migration, and chemo- or radio-resistance $[15,33,34,59,70,84,91$, 96]. Prolonged Nrf2 activation occurs when autophagy is dysregulated, either by deletion of $\operatorname{Atg} 5$ and $\operatorname{Atg} 7$, or when autophagosome-lysosome fusion is blocked by arsenic treatment, causing the formation of p62-Keap1 aggregates and Nrf2 activation [44]. Autophagy dysfunction and prolonged Nrf2 activation cause tissue damage, inflammation, fibrosis, and tumorigenesis. In the lungs, inducible disruption of $\operatorname{Atg} 7$ caused bronchiolar epithelial cell edema and hyperresponsiveness of the lungs to a cholinergic stimulus [40]. Interestingly, these $\operatorname{Atg} 7^{-1}$ cells had p62 accumulation and increased levels of Nrf2 target genes. On the other hand, basal or pharmacologically induced autophagy, and the subsequent activation of Nrf2, may constitute a good approach for lung protection. This mode of autophagy activation, much like the canonical activation of Nrf2, is oscillatory: activation of autophagy occurs after a stimulus (for example, oxidative stress or rapamycin administration) and elimination of the stimulus (restoration of redox homeostasis or drug metabolism and excretion) restores the pathway to basal levels. Therefore, this controlled dual Nrf2-autophagy activation maintains the cytoprotective properties of both pathways.

\section{Conclusion}

ALI/ARDS are lung dysfunction syndromes with high prevalence and mortality, mainly because no therapies against them have been approved. ALI can be induced directly or indirectly by oxidative stress. Airway and lung epithelial cells are protected from oxidative stress by the Nrf2 and autophagy pathways. While extensive research has demonstrated the importance of Nrf2 in the protection against ALI and other lung pathologies, the role of autophagy is still ambiguous, though sufficient evidence supports its role as protective. Controlled dual activation of Nrf2 and autophagy may prove to be a better treatment strategy than single pathway activation due to the existing crosstalk of these pathways and potential synergistic beneficial effects.

Acknowledgments This research was supported in part by the following National Institutes of Health grants: R01CA154377, R01ES015010, and R01ES023758 (DDZ); P01HL58064 (JGNG); HL60190, HL67841, and P01HL0101902 (SMB).

\section{Compliance with Ethical Standards}

Conflict of Interest The authors have no conflict of interest to disclose.

Human and Animal Rights and Informed Consent This article does not contain any studies with human or animal subjects performed by any of the authors.

\section{References}

1. Abel EL, Bubel JD, Simper MS, Powell L, McClellan SA, Andreeff $\mathrm{M}$, et al. Protection against 2-chloroethyl ethyl sulfide (CEES)-induced cytotoxicity in human keratinocytes by an inducer of the glutathione detoxification pathway. Toxicol Appl Pharmacol. 2011;255(2):176-83. doi:10.1016/j.taap.2011.06.012.

2. Aggarwal S, Dimitropoulou C, Lu Q, Black SM, Sharma S. Glutathione supplementation attenuates lipopolysaccharideinduced mitochondrial dysfunction and apoptosis in a mouse model of acute lung injury. Front Physiol. 2012;3:161. doi:10. 3389/fphys.2012.00161

3. Ashbaugh DG, Bigelow DB, Petty TL, Levine BE. Acute respiratory distress in adults. Lancet. 1967;2(7511):319-23.

4. Athale J, Ulrich A, Chou Macgarvey N, Bartz RR, Welty-Wolf KE, Suliman HB, et al. Nrf2 promotes alveolar mitochondrial biogenesis and resolution of lung injury in Staphylococcus aureus pneumonia in mice. Free Radic Biol Med. 2012;53(8):1584-94. doi:10.1016/j.freeradbiomed.2012.08.009.

5. Baird L, Lleres D, Swift S, Dinkova-Kostova AT. Regulatory flexibility in the Nrf2-mediated stress response is conferred by 
conformational cycling of the Keap1-Nrf2 protein complex. Proc Natl Acad Sci U S A. 2013;110(38):15259-64. doi:10.1073/pnas. 1305687110.

6. Beasley MB. The pathologist's approach to acute lung injury. Arch Pathol Lab Med. 2010;134(5):719-27. doi:10.1043/15432165-134.5.719.

7. Bernard GR, Artigas A, Brigham KL, Carlet J, Falke K, Hudson $\mathrm{L}$, et al. The American-European Consensus Conference on ARDS. Definitions, mechanisms, relevant outcomes, and clinical trial coordination. Am J Respir Crit Care Med. 1994;149(3 Pt 1): 818-24. doi:10.1164/ajrccm.149.3.7509706.

8. Bernard K, Hecker L, Luckhardt TR, Cheng G, Thannickal VJ. NADPH oxidases in lung health and disease. Antioxid Redox Signal. 2014;20(17):2838-53. doi:10.1089/ars.2013.5608.

9. Brune B, Dehne N, Grossmann N, Jung M, Namgaladze D, Schmid T, et al. Redox control of inflammation in macrophages. Antioxid Redox Signal. 2013;19(6):595-637. doi:10.1089/ars. 2012.4785.

10. Campos R, Shimizu MH, Volpini RA, de Braganca AC, Andrade $\mathrm{L}$, Lopes FD, et al. N-acetylcysteine prevents pulmonary edema and acute kidney injury in rats with sepsis submitted to mechanical ventilation. Am J Physiol Lung Cell Mol Physiol. 2012;302(7): L640-50. doi:10.1152/ajplung.00097.2011.

11. Castillo EF, Dekonenko A, Arko-Mensah J, Mandell MA, Dupont $\mathrm{N}$, Jiang $\mathrm{S}$, et al. Autophagy protects against active tuberculosis by suppressing bacterial burden and inflammation. Proc Natl Acad Sci U S A. 2012;109(46):E3168-76. doi:10.1073/pnas. 1210500109.

12. Chan K, Kan YW. Nrf2 is essential for protection against acute pulmonary injury in mice. Proc Natl Acad Sci U S A. 1999;96(22):12731-6

13. Chauhan S, Chauhan S, D'Cruz R, Faruqi S, Singh KK, Varma S, et al. Chemical warfare agents. Environ Toxicol Pharmacol. 2008;26(2):113-22. doi:10.1016/j.etap.2008.03.003.

14. Chen D, Ma T, Liu XW, Yang C, Liu Z. Rapamycin reverses paraquat-induced acute lung injury in a rat model through inhibition of NFkappaB activation. Int J Clin Exp Pathol. 2015;8(5): 4627-38.

15. Chen W, Sun Z, Wang XJ, Jiang T, Huang Z, Fang D, et al. Direct interaction between $\mathrm{Nrf} 2$ and $\mathrm{p} 21$ (Cip1/WAF1) upregulates the Nrf2-mediated antioxidant response. Mol Cell. 2009;34(6):66373. doi:10.1016/j.molcel.2009.04.029.

16. Chen ZH, Kim HP, Sciurba FC, Lee SJ, Feghali-Bostwick C, Stolz DB, et al. Egr-1 regulates autophagy in cigarette smoke-induced chronic obstructive pulmonary disease. PLoS One. 2008;3(10), e3316. doi:10.1371/journal.pone.0003316.

17. Chen ZH, Lam HC, Jin Y, Kim HP, Cao J, Lee SJ, et al. Autophagy protein microtubule-associated protein 1 light chain3B (LC3B) activates extrinsic apoptosis during cigarette smokeinduced emphysema. Proc Natl Acad Sci U S A. 2010;107(44): 18880-5. doi:10.1073/pnas.1005574107.

18. Cho HY. Genomic structure and variation of nuclear factor (erythroid-derived 2)-like 2. Oxidative Med Cell Longev. 2013;2013: 286524. doi:10.1155/2013/286524.

19. Cho HY, Gladwell W, Yamamoto M, Kleeberger SR. Exacerbated airway toxicity of environmental oxidant ozone in mice deficient in Nrf2. Oxidative Med Cell Longev. 2013;2013:254069. doi:10. 1155/2013/254069.

20. Cho HY, Jedlicka AE, Gladwell W, Marzec J, McCaw ZR, Bienstock RJ, et al. Association of Nrf2 polymorphism haplotypes with acute lung injury phenotypes in inbred strains of mice. Antioxid Redox Signal. 2015;22(4):325-38. doi:10.1089/ars. 2014.5942.

21. Cho HY, Jedlicka AE, Reddy SP, Kensler TW, Yamamoto M, Zhang LY, et al. Role of NRF2 in protection against hyperoxic lung injury in mice. Am J Respir Cell Mol Biol. 2002;26(2):17582. doi:10.1165/ajrcmb.26.2.4501.

22. Cho HY, Kleeberger SR. Noblesse oblige: NRF2 functions in the airways. Am J Respir Cell Mol Biol. 2014;50(5):844-7. doi:10. 1165/rcmb.2014-0116PS.

23. Cho HY, Kleeberger SR. Association of Nrf2 with airway pathogenesis: lessons learned from genetic mouse models. Arch Toxicol. 2015. doi:10.1007/s00204-015-1557-y.

24. Cho HY, Marzec J, Kleeberger SR. Functional polymorphisms in NRF2: implications for human disease. Free Radic Biol Med. 2015. doi:10.1016/j.freeradbiomed.2015.06.012.

25. Cho HY, Reddy SP, Kleeberger SR. Nrf2 defends the lung from oxidative stress. Antioxid Redox Signal. 2006;8(1-2):76-87. doi: 10.1089/ars.2006.8.76.

26. Choi AM, Ryter SW, Levine B. Autophagy in human health and disease. N Engl J Med. 2013;368(7):651-62.

27. Chowdhry S, Zhang Y, McMahon M, Sutherland C, Cuadrado A, Hayes JD. Nrf2 is controlled by two distinct beta-TrCP recognition motifs in its Neh6 domain, one of which can be modulated by GSK-3 activity. Oncogene. 2013;32(32):3765-81. doi:10.1038/ onc. 2012.388

28. Cordova EJ, Jimenez-Morales S, Centeno F, Martinez-Hernandez A, Martinez-Aguilar N, Del-Rio-Navarro BE, et al. NFE2L2 gene variants and susceptibility to childhood-onset asthma. Rev Invest Clin. 2011;63(4):407-11.

29. Dinkova-Kostova AT, Abramov AY. The emerging role of Nrf2 in mitochondrial function. Free Radic Biol Med. 2015. doi:10.1016/ j.freeradbiomed.2015.04.036.

30. Fan W, Tang Z, Chen D, Moughon D, Ding X, Chen S, et al. Keap1 facilitates p62-mediated ubiquitin aggregate clearance via autophagy. Autophagy. 2010;6(5):614-21. doi:10.4161/auto.6.5. 12189.

31. Francis RC, Vaporidi K, Bloch KD, Ichinose F, Zapol WM. Protective and detrimental effects of sodium sulfide and hydrogen sulfide in murine ventilator-induced lung injury. Anesthesiology. 2011;115(5):1012-21. doi:10.1097/ALN.0b013e31823306cf.

32. Harris J, Hartman M, Roche C, Zeng SG, O’Shea A, Sharp FA, et al. Autophagy controls IL-1beta secretion by targeting pro-IL1 beta for degradation. J Biol Chem. 2011;286(11):9587-97. doi: 10.1074/jbc.M110.202911.

33. Hast BE, Goldfarb D, Mulvaney KM, Hast MA, Siesser PF, Yan F, et al. Proteomic analysis of ubiquitin ligase KEAP1 reveals associated proteins that inhibit NRF2 ubiquitination. Cancer Res. 2013;73(7):2199-210. doi:10.1158/0008-5472.CAN-12-4400.

34. Hayes JD, McMahon M. NRF2 and KEAP1 mutations: permanent activation of an adaptive response in cancer. Trends Biochem Sci. 2009;34(4):176-88. doi:10.1016/j.tibs.2008.12.008.

35. Herridge MS, Tansey CM, Matte A, Tomlinson G, Diaz-Granados $\mathrm{N}$, Cooper A, et al. Functional disability 5 years after acute respiratory distress syndrome. N Engl J Med. 2011;364(14):1293-304. doi:10.1056/NEJMoa1011802.

36. Howard MD, Greineder CF, Hood ED, Muzykantov VR. Endothelial targeting of liposomes encapsulating SOD/catalase mimetic EUK-134 alleviates acute pulmonary inflammation. J Control Release. 2014;177:34-41. doi:10.1016/j.jconrel.2013. 12.035 .

37. Hu ZS, Gu ZL, Sun MN, Zhang K, Gao PH, Yang QW, et al. Ursolic acid improves survival and attenuates lung injury in septic rats induced by cecal ligation and puncture. J Surg Res. 2015;194(2):528-36. doi:10.1016/j.jss.2014.10.027.

38. Husari A, Khayat A, Bitar H, Hashem Y, Rizkallah A, Zaatari G, et al. Antioxidant activity of pomegranate juice reduces acute lung injury secondary to hyperoxia in an animal model. BMC Res Notes. 2014;7:664. doi:10.1186/1756-0500-7-664.

39. Ichimura Y, Waguri S, Sou YS, Kageyama S, Hasegawa J, Ishimura $\mathrm{R}$, et al. Phosphorylation of p62 activates the Keap1- 
Nrf2 pathway during selective autophagy. Mol Cell. 2013;51(5): 618-31. doi:10.1016/j.molcel.2013.08.003.

40. Inoue D, Kubo H, Taguchi K, Suzuki T, Komatsu M, Motohashi $\mathrm{H}$, et al. Inducible disruption of autophagy in the lung causes airway hyper-responsiveness. Biochem Biophys Res Commun. 2011;405(1):13-8. doi:10.1016/j.bbrc.2010.12.092.

41. Jain A, Lamark T, Sjottem E, Larsen KB, Awuh JA, Overvatn A, et al. p62/SQSTM1 is a target gene for transcription factor NRF2 and creates a positive feedback loop by inducing antioxidant response element-driven gene transcription. J Biol Chem. 2010;285(29):22576-91. doi:10.1074/jbc.M110.118976.

42. Jaramillo MC, Zhang DD. The emerging role of the Nrf2-Keap1 signaling pathway in cancer. Genes Dev. 2013;27(20):2179-91. doi:10.1101/gad.225680.113.

43. Ji L, Liu R, Zhang XD, Chen HL, Bai H, Wang X, et al. Nacetylcysteine attenuates phosgene-induced acute lung injury via up-regulation of Nrf2 expression. Inhal Toxicol. 2010;22(7):53542. doi:10.3109/08958370903525183.

44. Jiang T, Harder B, Vega MR, Wong PK, Chapman E, Zhang DD. p62 links autophagy and Nrf2 signaling. Free Radic Biol Med. 2015. doi:10.1016/j.freeradbiomed.2015.06.014.

45. Junkins RD, Shen A, Rosen K, McCormick C, Lin TJ. Autophagy enhances bacterial clearance during P. aeruginosa lung infection. PLoS One. 2013;8(8), e72263. doi:10.1371/journal.pone. 0072263.

46. Jurkuvenaite A, Benavides GA, Komarova S, Doran SF, Johnson M, Aggarwal S, et al. Upregulation of autophagy decreases chlorine-induced mitochondrial injury and lung inflammation. Free Radic Biol Med. 2015;85:83-94. doi:10.1016/j. freeradbiomed.2015.03.039.

47. Kensler TW, Wakabayashi N, Biswal S. Cell survival responses to environmental stresses via the Keap1-Nrf2-ARE pathway. Annu Rev Pharmacol Toxicol. 2007;47:89-116. doi:10.1146/annurev. pharmtox.46.120604.141046.

48. Kobayashi A, Kang MI, Okawa H, Ohtsuji M, Zenke Y, Chiba T, et al. Oxidative stress sensor Keap1 functions as an adaptor for Cul3-based E3 ligase to regulate proteasomal degradation of Nrf2. Mol Cell Biol. 2004;24(16):7130-9. doi:10.1128/MCB.24.16. 7130-7139.2004.

49. Komatsu M, Kurokawa H, Waguri S, Taguchi K, Kobayashi A, Ichimura $\mathrm{Y}$, et al. The selective autophagy substrate p62 activates the stress responsive transcription factor Nrf2 through inactivation of Keap1. Nat Cell Biol. 2010;12(3):213-23. doi:10.1038/ ncb2021.

50. Kroemer G, Marino G, Levine B. Autophagy and the integrated stress response. Mol Cell. 2010;40(2):280-93. doi:10.1016/j. molcel.2010.09.023

51. Lau A, Villeneuve NF, Sun Z, Wong PK, Zhang DD. Dual roles of Nrf2 in cancer. Pharmacol Res. 2008;58(5-6):262-70. doi:10. 1016/j.phrs.2008.09.003

52. Lau A, Wang XJ, Zhao F, Villeneuve NF, Wu T, Jiang T, et al. A noncanonical mechanism of Nrf2 activation by autophagy deficiency: direct interaction between Keap1 and p62. Mol Cell Biol. 2010;30(13):3275-85. doi:10.1128/MCB.00248-10.

53. Lau A, Zheng Y, Tao S, Wang H, Whitman SA, White E, et al. Arsenic inhibits autophagic flux, activating the Nrf2-Keap1 pathway in a p62-dependent manner. Mol Cell Biol. 2013;33(12): 2436-46. doi:10.1128/MCB.01748-12.

54. Lee SJ, Ryter SW, Xu JF, Nakahira K, Kim HP, Choi AM, et al. Carbon monoxide activates autophagy via mitochondrial reactive oxygen species formation. Am J Respir Cell Mol Biol. 2011;45(4):867-73. doi:10.1165/rcmb.2010-0352OC.

55. Levine B, Liu R, Dong X, Zhong Q. Beclin orthologs: integrative hubs of cell signaling, membrane trafficking, and physiology. Trends Cell Biol. 2015. doi:10.1016/j.tcb.2015.05.004.
56. Lingaraju MC, Pathak NN, Begum J, Balaganur V, Bhat RA, Ram $\mathrm{M}$, et al. Betulinic acid negates oxidative lung injury in surgical sepsis model. J Surg Res. 2015;193(2):856-67. doi:10.1016/j.jss. 2014.09.008.

57. Lo S, Yuan SS, Hsu C, Cheng YJ, Chang YF, Hsueh HW, et al. Lc3 over-expression improves survival and attenuates lung injury through increasing autophagosomal clearance in septic mice. Ann Surg. 2013;257(2):352-63. doi:10.1097/SLA.0b013e318269d0e2.

58. Lorne E, Zhao X, Zmijewski JW, Liu G, Park YJ, Tsuruta Y, et al. Participation of mammalian target of rapamycin complex 1 in Toll-like receptor 2- and 4-induced neutrophil activation and acute lung injury. Am J Respir Cell Mol Biol. 2009;41(2):237-45. doi: 10.1165/rcmb.2008-02900C.

59. Ma J, Cai H, Wu T, Sobhian B, Huo Y, Alcivar A, et al. PALB2 interacts with KEAP1 to promote NRF2 nuclear accumulation and function. Mol Cell Biol. 2012;32(8):1506-17. doi:10.1128/MCB. 06271-11.

60. Malaviya R, Laskin JD, Laskin DL. Oxidative stress-induced autophagy: role in pulmonary toxicity. Toxicol Appl Pharmacol. 2014;275(2):145-51. doi:10.1016/j.taap.2013.12.022.

61. Malaviya R, Sunil VR, Cervelli J, Anderson DR, Holmes WW, Conti ML, et al. Inflammatory effects of inhaled sulfur mustard in rat lung. Toxicol Appl Pharmacol. 2010;248(2):89-99. doi:10. 1016/j.taap.2010.07.018.

62. Mamary AJ, Kondapaneni S, Vance GB, Gaughan JP, Martin UJ, Criner GJ. Survival in patients receiving prolonged ventilation: factors that influence outcome. Clin Med Insights Circ Respir Pulm Med. 2011;5:17-26. doi:10.4137/CCRPM.S6649.

63. Marzec JM, Christie JD, Reddy SP, Jedlicka AE, Vuong H, Lanken PN, et al. Functional polymorphisms in the transcription factor NRF2 in humans increase the risk of acute lung injury. FASEB J. 2007;21(9):2237-46. doi:10.1096/fj.06-7759com.

64. Matthay MA, Ware LB, Zimmerman GA. The acute respiratory distress syndrome. J Clin Investig. 2012;122(8):2731-40. doi:10. 1172/Jci60331.

65. Matute-Bello G, Frevert CW, Martin TR. Animal models of acute lung injury. Am J Physiol Lung Cell Mol Physiol. 2008;295(3): L379-99. doi:10.1152/ajplung.00010.2008.

66. McGovern T, Day BJ, White CW, Powell WS, Martin JG. AEOL10150: a novel therapeutic for rescue treatment after toxic gas lung injury. Free Radic Biol Med. 2011;50(5):602-8. doi:10. 1016/j.freeradbiomed.2010.12.001.

67. Mittal M, Siddiqui MR, Tran K, Reddy SP, Malik AB. Reactive oxygen species in inflammation and tissue injury. Antioxid Redox Signal. 2014;20(7):1126-67. doi:10.1089/ars.2012.5149.

68. Mochizuki M, Ishii Y, Itoh K, Iizuka T, Morishima Y, Kimura T, et al. Role of 15-deoxy delta(12,14) prostaglandin J2 and Nrf2 pathways in protection against acute lung injury. Am J Respir Crit Care Med. 2005;171(11):1260-6. doi:10.1164/rccm. 200406-755OC.

69. Nakahira K, Haspel JA, Rathinam VA, Lee SJ, Dolinay T, Lam $\mathrm{HC}$, et al. Autophagy proteins regulate innate immune responses by inhibiting the release of mitochondrial DNA mediated by the NALP3 inflammasome. Nat Immunol. 2011;12(3):222-30. doi: 10.1038/ni.1980.

70. Ooi A, Dykema K, Ansari A, Petillo D, Snider J, Kahnoski R, et al. CUL3 and NRF2 mutations confer an NRF2 activation phenotype in a sporadic form of papillary renal cell carcinoma. Cancer Res. 2013;73(7):2044-51. doi:10.1158/0008-5472.CAN-123227.

71. Papaiahgari S, Yerrapureddy A, Reddy SR, Reddy NM, Dodd OJ, Crow MT, et al. Genetic and pharmacologic evidence links oxidative stress to ventilator-induced lung injury in mice. Am J Respir Crit Care Med. 2007;176(12):1222-35. doi:10.1164/rccm. 200701-0600C. 
72. Pita R, Marco-Contelles J, Ramos E, Del Pino J, Romero A. Toxicity induced by chemical warfare agents: insights on the protective role of melatonin. Chem Biol Interact. 2013;206(2):134 42. doi:10.1016/j.cbi.2013.09.001.

73. Rada P, Rojo AI, Chowdhry S, McMahon M, Hayes JD, Cuadrado A. $\mathrm{SCF} /\{$ beta\}-TrCP promotes glycogen synthase kinase 3dependent degradation of the Nrf2 transcription factor in a Keap1-independent manner. Mol Cell Biol. 2011;31(6):1121-33. doi:10.1128/MCB.01204-10.

74. Rada P, Rojo AI, Evrard-Todeschi N, Innamorato NG, Cotte A, Jaworski T, et al. Structural and functional characterization of $\mathrm{Nrf} 2$ degradation by the glycogen synthase kinase 3/beta-TrCP axis. Mol Cell Biol. 2012;32(17):3486-99. doi:10.1128/MCB.00180-12.

75. Ravikumar B, Sarkar S, Davies JE, Futter M, Garcia-Arencibia M, Green-Thompson ZW, et al. Regulation of mammalian autophagy in physiology and pathophysiology. Physiol Rev. 2010;90(4): 1383-435. doi:10.1152/physrev.00030.2009.

76. Reddy NM, Kleeberger SR, Kensler TW, Yamamoto M, Hassoun PM, Reddy SP. Disruption of Nrf2 impairs the resolution of hyperoxia-induced acute lung injury and inflammation in mice. J Immunol. 2009;182(11):7264-71. doi:10.4049/jimmunol. 0804248 .

77. Reddy NM, Potteti HR, Mariani TJ, Biswal S, Reddy SP. Conditional deletion of Nrf2 in airway epithelium exacerbates acute lung injury and impairs the resolution of inflammation. Am J Respir Cell Mol Biol. 2011;45(6):1161-8. doi:10.1165/ rcmb.2011-0144OC.

78. Reddy NM, Suryanaraya V, Yates MS, Kleeberger SR, Hassoun PM, Yamamoto M, et al. The triterpenoid CDDO-imidazolide confers potent protection against hyperoxic acute lung injury in mice. Am J Respir Crit Care Med. 2009;180(9):867-74. doi:10. 1164/rccm.200905-0670OC.

79. Reddy NM, Suryanarayana V, Kalvakolanu DV, Yamamoto M, Kensler TW, Hassoun PM, et al. Innate immunity against bacterial infection following hyperoxia exposure is impaired in NRF2deficient mice. J Immunol. 2009;183(7):4601-8. doi:10.4049/ jimmunol.0901754.

80. Ricciotti E, FitzGerald GA. Prostaglandins and inflammation. Arterioscler Thromb Vasc Biol. 2011;31(5):986-1000. doi:10. 1161/ATVBAHA.110.207449.

81. Riley BE, Kaiser SE, Shaler TA, Ng AC, Hara T, Hipp MS, et al. Ubiquitin accumulation in autophagy-deficient mice is dependent on the Nrf2-mediated stress response pathway: a potential role for protein aggregation in autophagic substrate selection. J Cell Biol. 2010;191(3):537-52. doi:10.1083/jcb.201005012.

82. Rubenfeld GD, Caldwell E, Peabody E, Weaver J, Martin DP, Neff $\mathrm{M}$, et al. Incidence and outcomes of acute lung injury. $\mathrm{N}$ Engl J Med. 2005;353(16):1685-93. doi:10.1056/ NEJMoa050333.

83. Ryter SW, Choi AM. Autophagy in the lung. Proc Am Thorac Soc. 2010;7(1):13-21. doi:10.1513/pats.200909-101JS.

84. Shibata T, Ohta T, Tong KI, Kokubu A, Odogawa R, Tsuta K, et al. Cancer related mutations in NRF2 impair its recognition by Keap1-Cul3 E3 ligase and promote malignancy. Proc Natl Acad Sci U S A. 2008;105(36):13568-73. doi:10.1073/pnas. 0806268105 .

85. Shohrati M, Karimzadeh I, Saburi A, Khalili H, Ghanei M. The role of $\mathrm{N}$-acetylcysteine in the management of acute and chronic pulmonary complications of sulfur mustard: a literature review. Inhal Toxicol. 2014;26(9):507-23. doi:10.3109/08958378.2014. 920439.

86. Slutsky AS. Ventilator-induced lung injury: from barotrauma to biotrauma. Respir Care. 2005;50(5):646-59.

87. Sun Z, Wu T, Zhao F, Lau A, Birch CM, Zhang DD. KPNA6 (Importin \{alpha\}7)-mediated nuclear import of Keap1 represses the Nrf2-dependent antioxidant response. Mol Cell Biol. 2011;31(9):1800-11. doi:10.1128/MCB.05036-11.

88. Sunil VR, Patel-Vayas K, Shen J, Laskin JD, Laskin DL. Classical and alternative macrophage activation in the lung following ozone-induced oxidative stress. Toxicol Appl Pharmacol. 2012;263(2):195-202. doi:10.1016/j.taap.2012.06.009.

89. Taguchi K, Fujikawa N, Komatsu M, Ishii T, Unno M, Akaike T, et al. Keap1 degradation by autophagy for the maintenance of redox homeostasis. Proc Natl Acad Sci U S A. 2012;109(34): 13561-6. doi:10.1073/pnas.1121572109.

90. Tao S, Rojo de la Vega M, Quijada H, Wondrak GT, Wang T, Garcia JGN et al. Bixin protects mice against ventilationinduced lung injury in an NRF2-dependent manner. Sci Rep. 2016;6:18760.

91. Tao S, Wang S, Moghaddam SJ, Ooi A, Chapman E, Wong PK, et al. Oncogenic KRAS confers chemoresistance by upregulating NRF2. Cancer Res. 2014;74(24):7430-41. doi:10.1158/00085472.CAN-14-1439.

92. Tong KI, Kobayashi A, Katsuoka F, Yamamoto M. Two-site substrate recognition model for the Keap1-Nrf2 system: a hinge and latch mechanism. Biol Chem. 2006;387(10-11):1311-20. doi:10. 1515/BC.2006.164.

93. Tong KI, Padmanabhan B, Kobayashi A, Shang C, Hirotsu Y, Yokoyama S, et al. Different electrostatic potentials define ETGE and DLG motifs as hinge and latch in oxidative stress response. Mol Cell Biol. 2007;27(21):7511-21. doi:10.1128/ MCB.00753-07.

94. Villeneuve NF, Lau A, Zhang DD. Regulation of the Nrf2-Keap1 antioxidant response by the ubiquitin proteasome system: an insight into cullin-ring ubiquitin ligases. Antioxid Redox Signal. 2010;13(11):1699-712. doi:10.1089/ars.2010.3211.

95. Walkey AJ, Summer R, Ho V, Alkana P. Acute respiratory distress syndrome: epidemiology and management approaches. Clin Epidemiol. 2012;4:159-69. doi:10.2147/CLEP.S28800.

96. Wang R, An J, Ji F, Jiao H, Sun H, Zhou D. Hypermethylation of the Keap1 gene in human lung cancer cell lines and lung cancer tissues. Biochem Biophys Res Commun. 2008;373(1):151-4.

97. Warabi E, Takabe W, Minami T, Inoue K, Itoh K, Yamamoto M, et al. Shear stress stabilizes NF-E2-related factor 2 and induces antioxidant genes in endothelial cells: role of reactive oxygen/ nitrogen species. Free Radic Biol Med. 2007;42(2):260-9. doi: 10.1016/j.freeradbiomed.2006.10.043.

98. Ward HE, Nicholas TE. Alveolar type I and type II cells. Aust N Z J Med. 1984;14(5 Suppl 3):731-4.

99. Ware LB, Matthay MA. The acute respiratory distress syndrome. N Engl J Med. 2000;342(18):1334-49. doi:10.1056/ NEJM200005043421806.

100. Wilson MR, Takata M. Inflammatory mechanisms of ventilatorinduced lung injury: a time to stop and think? Anaesthesia. 2013;68(2):175-8. doi:10.1111/anae.12085.

101. Wright BJ. Lung-protective ventilation strategies and adjunctive treatments for the emergency medicine patient with acute respiratory failure. Emerg Med Clin North Am. 2014;32(4):871-87. doi: 10.1016/j.emc.2014.07.012.

102. Wu T, Zhao F, Gao B, Tan C, Yagishita N, Nakajima T, et al. Hrd1 suppresses Nrf2-mediated cellular protection during liver cirrhosis. Genes Dev. 2014;28(7):708-22. doi:10.1101/gad.238246.114.

103. Yamamoto Y, Sousse LE, Enkhbaatar P, Kraft ER, Deyo DJ, Wright CL, et al. Gamma-tocopherol nebulization decreases oxidative stress, arginase activity, and collagen deposition after burn and smoke inhalation in the ovine model. Shock. 2012;38(6):6716. doi:10.1097/SHK.0b013e3182758759.

104. Ye S, Lowther S, Stambas J. Inhibition of reactive oxygen species production ameliorates inflammation induced by influenza A viruses via upregulation of SOCS1 and SOCS3. J Virol. 2015;89(5): 2672-83. doi:10.1128/JVI.03529-14. 
105. Yen YT, Yang HR, Lo HC, Hsieh YC, Tsai SC, Hong CW, et al. Enhancing autophagy with activated protein $\mathrm{C}$ and rapamycin protects against sepsis-induced acute lung injury. Surgery. 2013;153(5):689-98. doi:10.1016/j.surg.2012.11.021.

106. Yilmaz MZ, Guzel A, Torun AC, Okuyucu A, Salis O, Karli R, et al. The therapeutic effects of anti-oxidant and anti-inflammatory drug quercetin on aspiration-induced lung injury in rats. J Mol Histol. 2014;45(2):195-203. doi:10. 1007/s10735-013-9542-3.

107. Yuan KF, Huang CH, Fox J, Laturnus D, Carlson E, Zhang BJ, et al. Autophagy plays an essential role in the clearance of Pseudomonas aeruginosa by alveolar macrophages. J Cell Sci. 2012;125(2):507-15. doi:10.1242/jcs.094573.

108. Zhang DD. Mechanistic studies of the Nrf2-Keap1 signaling pathway. Drug Metab Rev. 2006;38(4):769-89. doi:10.1080/ 03602530600971974.

109. Zhang DD, Hannink M. Distinct cysteine residues in Keap1 are required for Keap1-dependent ubiquitination of $\mathrm{Nrf2}$ and for stabilization of Nrf2 by chemopreventive agents and oxidative stress. Mol Cell Biol. 2003;23(22):8137-51.

110. Zhang DD, Lo SC, Cross JV, Templeton DJ, Hannink M. Keap1 is a redox-regulated substrate adaptor protein for a Cul3-dependent ubiquitin ligase complex. Mol Cell Biol. 2004;24(24):10941-53. doi:10.1128/MCB.24.24.10941-10953.2004.

111. Zhang T, Qi Y, Liao M, Xu M, Bower K, Frank J, et al. Autophagy is a cell self-protective mechanism against arsenic-induced cell transformation. Toxicol Sci. 2012. doi:10.1093/toxsci/kfs240.

112. Zhao WC, Zhou SL, Yao WF, Gan XL, Su GJ, Yuan DD, et al. Propofol prevents lung injury after intestinal ischemia-reperfusion by inhibiting the interaction between mast cell activation and oxidative stress. Life Sci. 2014;108(2):80-7. doi:10.1016/j.lfs.2014.05.009.

113. Zhu L, Pi J, Wachi S, Andersen ME, Wu R, Chen Y. Identification of Nrf2-dependent airway epithelial adaptive response to proinflammatory oxidant-hypochlorous acid challenge by transcription profiling. Am J Physiol Lung Cell Mol Physiol. 2008;294(3): L469-77. doi:10.1152/ajplung.00310.2007. 